H. Witała@ • J. Golak • R. Skibiński • K. Topolnicki •

E. Epelbaum - K. Hebeler • H. Kamada - H. Krebs •

U.-G. Meißner · A. Nogga

\title{
Application of Semilocal Coordinate-Space Regularized Chiral Forces to Elastic Nd Scattering and Breakup
}

\author{
Dedicated to Professor Ludwig Dmitriyevich Faddeev.
}

Received: 4 November 2018 / Accepted: 28 January 2019 / Published online: 25 February 2019

(C) The Author(s) 2019

\begin{abstract}
We solve three-nucleon (3N) Faddeev equations with nucleon-nucleon (NN) and three-nucleon forces (3NF) derived consistently in the framework of chiral perturbation theory, taking the semilocal coordinate-space regularized chiral $\mathrm{N}^{4} \mathrm{LO} N \mathrm{~N}$ potential supplemented by the chiral $\mathrm{N}^{2} \mathrm{LO} 3 \mathrm{NF}$ regularized in the same way. Based on these solutions the nucleon-deuteron (Nd) elastic scattering and deuteron breakup reactions are studied. We checked that the elastic Nd scattering cross section can be used as an alternative observable to the doublet neutron-deuteron (nd) scattering length to fix, together with the ${ }^{3} \mathrm{H}$ binding energy, the strengths of the contact terms of the $\mathrm{N}^{2} \mathrm{LO} 3 \mathrm{NF}$. We investigated the predicted $3 \mathrm{NF}$ effects in $3 \mathrm{~N}$ continuum reactions putting an emphasis on the $A_{y}$ puzzle in low energy nd elastic scattering and on cross sections in
\end{abstract}

This article belongs to the Topical Collection "Ludwig Faddeev Memorial Issue".

H. Witała $(\bowtie) \cdot$ J. Golak · R. Skibiński · K. Topolnicki

M. Smoluchowski Institute of Physics, Jagiellonian University, 30348 Kraków, Poland

E-mail: henryk.witala@uj.edu.pl

E. Epelbaum $\cdot$ H. Krebs

Institut für Theoretische Physik II, Ruhr-Universität Bochum, 44780 Bochum, Germany

K. Hebeler

Institut für Kernphysik, Technische Universität Darmstadt, 64289 Darmstadt, Germany

K. Hebeler

Extreme Matter Institute EMMI, GSI Helmholtzzentrum für Schwerionenforschung GmbH, 64291 Darmstadt, Germany

H. Kamada

Department of Physics, Faculty of Engineering, Kyushu Institute of Technology, Kitakyushu 804-8550, Japan

U.-G. Meißner

Helmholtz-Institut für Strahlen- und Kernphysik and Bethe Center for Theoretical Physics, Universität Bonn, 53115 Bonn, Germany

U.-G. Meißner

Institute for Advanced Simulation, Institut für Kernphysik, Jülich Center for Hadron Physics, 52425 Jülich, Germany

U.-G. Meißner

JARA - High Performance Computing Forschungszentrum Jülich, 52425 Jülich, Germany

A. Nogga

Institut für Kernphysik, Institute for Advanced Simulation and Jülich Center for Hadron Physics, Forschungszentrum Jülich, 52425 Jülich, Germany 
the symmetrical-space-star and quasi-free-scattering breakup configurations. We found that the $\mathrm{N}^{2} \mathrm{LO} 3 \mathrm{NF}$ provides effects comparable to those of standard, (semi-)phenomenological $2 \pi$-exchange Tucson-Melbourne 3NF.

\section{Introduction}

Since their advent numerically exact three-nucleon continuum Faddeev calculations of the elastic Nd scattering and deuteron breakup reactions have become a powerful tool to test modern models of the nuclear forces. After establishing a clear underbinding of ${ }^{3} \mathrm{H}$ and ${ }^{3} \mathrm{He}$ and revealing that this underbinding can be removed by adding a $3 \mathrm{NF}$ to a $3 \mathrm{~N}$ Hamiltonian, the question about the importance of a $3 \mathrm{NF}$ has become one of the main topics of $3 \mathrm{~N}$ system studies. With availability of a new generation of (semi-)phenomenological nucleon-nucleon interactions such as AV18 [1], CDBonn [2], Nijm1 and Nijm2 [3], which describe existing NN data with very high precision, these investigations received a new impetus. Comparison of theoretical predictions based on such potentials with precise Nd elastic scattering and breakup data in a wide range of incoming nucleon energies enabled to find out clear-cut discrepancies between theory and data allowing thus to reveal regions of large 3NF's effects. However, the models of 3NF's used in these studies [4], mainly of the $2 \pi$-exchange character, such as Tucson-Melbourne (TM99) [5] and Urbana IX [6], which are derived independently from the applied NN interactions, prevented arriving at unambiguous conclusions. For numerous observables these two models of 3NF's combined with the same NN potential led to different predictions, not supported by existing data [4], indicating that these models lack some important contributions.

With the advent of chiral perturbation theory (ChPT) approach it became possible to construct consistent two- and many-body nuclear forces which incorporate all possible contributions up to a given order of chiral expansion. High precision NN interactions up to the fifth order of chiral expansion $\left(\mathrm{N}^{4} \mathrm{LO}\right)$ have been developed which describe very precisely NN data below the pion production threshold [7-9]. In these first versions of chiral forces a nonlocal regularization in momentum space was applied to remove large-momentum contributions by employing a multiplicative factor $e^{-\left(p^{2} / \Lambda^{2}\right)^{n}}$ with some value of a cut-off regulator parameter $\Lambda[8,9]$. Such a regularization led, for small values of $\Lambda$, to a deterioration of the long-range behaviour of the NN potential and to problems when applying these forces to Nd elastic scattering at higher energies [10]. To avoid these problems a new method of regularization was proposed in [11,12]. It made use of a local regularization of the pion exchange contributions. The resulting semilocal coordinate-space regularized (SCS) chiral potentials contain the long-range part of the NN force predicted in a parameter-free way. First applications of these NN potentials to elastic $\mathrm{Nd}$ scattering and to the nucleon induced deuteron breakup reaction were described in $[13,14]$. Semilocal momentum-space regularized chiral potentials at fifth order are presented in Ref. [15].

Starting from $\mathrm{N}^{2} \mathrm{LO}$ a $3 \mathrm{NF}$ appears in a $3 \mathrm{~N}$ Hamiltonian [16]. Before applying such a Hamiltonian to structure and reaction calculations one must fix two free parameters of this $\mathrm{N}^{2} \mathrm{LO} 3 \mathrm{NF}$, namely the strength parameters $c_{D}$ and $c_{E}$ of the leading $1 \pi$-contact and the three-nucleon-contact terms, respectively. In the present study we explore and compare two different approaches to fix these parameters by looking at predictions for the binding energy of ${ }^{3} \mathrm{H}$ and one additional $3 \mathrm{~N}$ observable, obtained with $\mathrm{N}^{4} \mathrm{LO} \mathrm{NN}$ potential supplemented by a $\mathrm{N}^{2} \mathrm{LO} 3 \mathrm{NF}$ regularized in the same way. In the first approach as an additional observable the doublet nd scattering length is used while in the second case the elastic Nd scattering cross sections are applied. We investigate also $3 \mathrm{NF}$ effects predicted with such a combination of $2 \mathrm{~N}$ and $3 \mathrm{~N}$ forces for different $3 \mathrm{~N}$ continuum observables and compare them to the results obtained with the standard (semi-)phenomenological $2 \mathrm{~N}$ and $3 \mathrm{~N}$ interactions. In our previous investigations with SCS chiral forces $[13,14,17]$ we estimated the truncation errors of the chiral expansion following the algorithm formulated in Ref. [11]. In the present paper we do not show truncation errors and refer to Ref. [17] where the theoretical uncertainties for complete $\mathrm{N}^{2} \mathrm{LO}$ calculation, with $\mathrm{N}^{2} \mathrm{LO} \mathrm{NN}$ and $\mathrm{N}^{2} \mathrm{LO} 3 \mathrm{NF}$ combination, are shown.

The paper is organized as follows: in Sect. 2 we briefly describe how we solve $3 \mathrm{~N}$ bound and scattering Faddeev equations. Also two methods of fixing $c_{D}$ and $c_{E}$ parameters are presented together with results obtained when applying them. A discussion of results for Nd elastic scattering follows in Sect. 3 and for chosen complete breakup configurations in Sect. 4. We summarize and conclude in Sect. 5.

\section{Theoretical Formalism and Determination of $c_{D}$ and $c_{E}$}

Neutron-deuteron (nd) scattering with nucleons interacting through a two-body interaction $v_{N N}$ and a $3 \mathrm{NF}$ $V_{123}=V^{(1)}+V^{(2)}+V^{(3)}$, is described in terms of a breakup operator $T$ satisfying the Faddeev-type integral 
equation [18-20]

$$
\begin{aligned}
T|\phi\rangle= & t P|\phi\rangle+\left(1+t G_{0}\right) V^{(1)}(1+P)|\phi\rangle+t P G_{0} T|\phi\rangle \\
& +\left(1+t G_{0}\right) V^{(1)}(1+P) G_{0} T|\phi\rangle .
\end{aligned}
$$

The two-nucleon $t$-matrix $t$ is the solution of the Lippmann-Schwinger equation with the interaction $v_{N N} . V^{(1)}$ is the part of a $3 \mathrm{NF}$ which is symmetric under the interchange of nucleons 2 and 3 . The permutation operator $P=P_{12} P_{23}+P_{13} P_{23}$ is given in terms of the transposition operators, $P_{i j}$, which interchange nucleons $i$ and $j$. The incoming state $|\phi\rangle=\left|\mathbf{q}_{0}\right\rangle\left|\phi_{d}\right\rangle$ describes the free nd motion with the relative momentum $\mathbf{q}_{0}$ and $\left|\phi_{d}\right\rangle$ is the deuteron wave function. Finally, $G_{0}$ is the resolvent of the three-body center of mass kinetic energy. The amplitude for elastic scattering leading to the corresponding two-body final state $\left|\phi^{\prime}\right\rangle$ with the relative momentum $\mathbf{q}_{0}^{\prime}$ is then given by $[19,20]$

$$
\begin{aligned}
\left\langle\phi^{\prime}|U| \phi\right\rangle= & \left\langle\phi^{\prime}\left|P G_{0}^{-1}\right| \phi\right\rangle+\left\langle\phi^{\prime}|P T| \phi\right\rangle+\left\langle\phi^{\prime}\left|V^{(1)}(1+P)\right| \phi\right\rangle \\
& +\left\langle\phi^{\prime}\left|V^{(1)}(1+P) G_{0} T\right| \phi\right\rangle,
\end{aligned}
$$

while for the breakup reaction one has

$$
\left\langle\phi_{0}^{\prime}\left|U_{0}\right| \phi\right\rangle=\left\langle\phi_{0}^{\prime}|(1+P) T| \phi\right\rangle
$$

where $\left|\phi_{0}^{\prime}\right\rangle$ is the free three-body breakup channel state.

As a NN interaction we took the semi-locally regularized $\mathrm{N}^{4} \mathrm{LO}$ chiral potential of Refs. $[11,12]$ with the regulator $R=0.9 \mathrm{fm}$ alone or combined with the chiral $\mathrm{N}^{2} \mathrm{LO} 3 \mathrm{NF}$, regularized with the same regulator [16,21]. We additionally regularized matrix elements of that $3 \mathrm{NF}$ by multiplying it with a nonlocal regulator $f(p, q)=$ $\exp \left\{-\left(p^{2}+\frac{3}{4} q^{2}\right)^{3} / \Lambda^{6}\right\}$ with a large cut-off value $\Lambda=1000 \mathrm{MeV}$. This additional regulator is applied to the $3 \mathrm{NF}$ matrix elements only for technical reasons. Namely, the practical calculations of the matrix elements of the local $3 \mathrm{NF}$ involve the evaluation of convolution integrals whose calculation becomes numerically unstable at very large momenta. The value of the cutoff scale $\Lambda$ is chosen sufficiently large so that low-energy physics is not affected by this additional regulator. In fact, we have checked explicitly that the effects of this regulator for the chosen cutoff value are negligible in three-body bound state and scattering calculations. Since we will compare $3 \mathrm{~N}$ scattering predictions to proton-deuteron (pd) data as a neutron-neutron (nn) force we took the proton-proton (pp) version of that particular NN interaction (with the Coulomb force subtracted).

The nuclear Hamiltonian with a $3 \mathrm{NF}$ at $\mathrm{N}^{2} \mathrm{LO}$ is fixed by specifying the values of the $\mathrm{LECs} c_{D}$ and $c_{E}$ which parametrize the strengths of the leading $1 \pi$-contact and the three-nucleon-contact terms. To determine them we follow the approach of Ref. [16] and use the experimental triton binding energy $E\left({ }^{3} H\right)$ and an additional $3 \mathrm{~N}$ observable sensitive to the action of the $3 \mathrm{NF}$ as two quantities from which $c_{D}$ and $c_{E}$ are obtained. The procedure can be divided into two steps. First, the dependence of $E\left({ }^{3} H\right)$ on $c_{E}$ for a given value of $c_{D}$ is determined. The requirement to reproduce the experimental value of the triton binding energy yields a set of pairs $\left(c_{D}, c_{E}\right)$. To find this set we compute the ${ }^{3} \mathrm{H}$ wave function using the method described in [22], where the full triton wave function $|\Psi\rangle=(1+P)|\psi\rangle$ is given in terms of its Faddeev component $|\psi\rangle$, which fulfills the Faddeev equation

$$
|\psi\rangle=G_{0} t P|\psi\rangle+\left(1+G_{0} t\right) G_{0} V^{(1)}(1+P)|\psi\rangle
$$

When solving Eq. (4) we used nn and neutron-proton (np) versions of the $\mathrm{N}^{4} \mathrm{LO}$ chiral potential with the regulator $R=0.9 \mathrm{fm}$. In Fig. 1 (upper part) the dashed (black) line shows the $\left(c_{D}, c_{E}\right)$ values which reproduce the experimental binding energy of ${ }^{3} \mathrm{H}$.

These $\left(c_{D}, c_{E}\right)$ values are then used in the calculations of the second observable, which allows us to find out the $\left(c_{D}, c_{E}\right)$ pair describing both observables simultaneously. Using the triton binding energy and the nd doublet scattering length yields one possibility to fix the parameters in the $3 \mathrm{NF}$ but probably not the optimal one due to the strong correlation between these two observables [17,23]. The doublet scattering length ${ }^{2} a_{n d}$ is calculated using the $\left(c_{D}, c_{E}\right)$ pairs from the upper part of Fig. 1. To this end, we solve the Faddeev equation (1) for the auxiliary state $T|\phi\rangle$ at zero incoming energy [24] with the same interactions as for ${ }^{3} \mathrm{H}$. The requirement to reproduce, in addition to the binding energy of ${ }^{3} \mathrm{H}$, also the nd doublet scattering length leads 


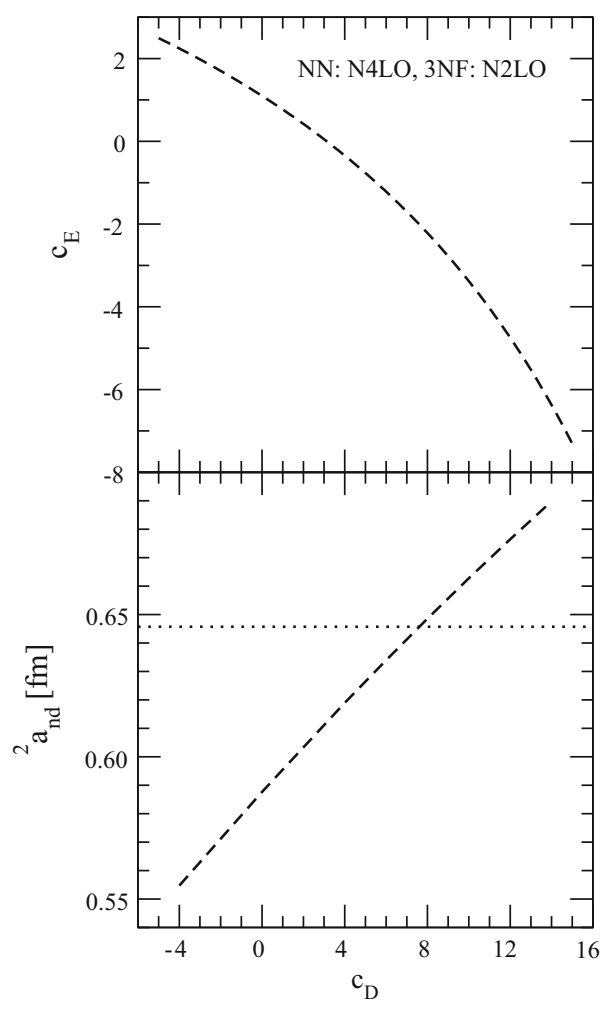

Fig. 1 Upper part: a correlation function for the strengths $c_{D}$ and $c_{E}$ of the $\mathrm{N}^{2} \mathrm{LO} 3 \mathrm{NF}$ contact terms. The ${ }^{3} \mathrm{H}$ experimental binding energy is reproduced by combining the $\mathrm{N}^{4} \mathrm{LO}$ SCS chiral NN potential with a $\mathrm{N}^{2} \mathrm{LO} 3 \mathrm{NF}$ whose strengths $c_{D}$ and $c_{E}$ lie on the dashed line. In the lower part the corresponding doublet nd scattering lengths ${ }^{2} a_{n d}$ are shown by the dashed line. The dotted line is the experimental value ${ }^{2} a_{n d}^{\exp }=0.645(7) \mathrm{fm}$ [25]. The regulator $R=0.9 \mathrm{fm}$ has been used

to the values $c_{D}=7.51 \pm 0.10$ and $c_{E}=1.79 \pm 0.05$ for the used combination of $\mathrm{N}^{4} \mathrm{LO} \mathrm{NN}$ and $\mathrm{N}^{2} \mathrm{LO} 3 \mathrm{~N}$ forces. The uncertainties of $c_{D}$ and $c_{E}$ result from the experimental uncertainty of the doublet nd scattering length [25].

We refer the reader to Refs. $[19,20,26]$ for a general overview of $3 \mathrm{~N}$ scattering and for more details on the practical implementation of the Faddeev equations. In order to provide convergent predictions we solved Eq. (1) taking into account all partial wave states with the total $2 \mathrm{~N}$ angular momentum $j \leq j_{\max }=5$ and the total $3 \mathrm{~N}$ angular momentum $J \leq J_{\max }=25 / 2$. The $3 \mathrm{NF}$ was included for $J \leq 7 / 2$.

It is important to address the question of uniqueness of our approach to determine the constants $c_{D}$ and $c_{E}$. To this end, we checked how taking instead of ${ }^{2} a_{n d}$ a different Nd observable, namely the elastic Nd scattering cross section, would influence the determination of $c_{D}$ and $c_{E}$. The low-energy elastic nd and pd scattering cross section is an observable which is well described by standard theory without the inclusion of 3NF's [27]. It turns out that when increasing the incoming nucleon laboratory energy, starting from $\approx 60 \mathrm{MeV}$, clear discrepancies between theory and data develop and pure $\mathrm{NN}$ potential predictions underestimate the data in the region of the cross section minimum up to backward scattering angles [28]. Thus it seems reasonable that the cross section in that angular range could be used to determine the constants $c_{D}$ and $c_{E}$. Since at larger energies higher orders of chiral expansion become important and a 3NF gets additional contributions, the procedure should be applied at energies where the discrepancies start to appear. Therefore we used three precise pd data sets taken by different groups, one at $E=65 \mathrm{MeV}$ from Ref. [29], second at $E=70 \mathrm{MeV}$ from Ref. [30], and third at $E=135 \mathrm{MeV}$ from Ref. [30], and performed $\chi^{2}$ fits of theory to data using four $\left(c_{D}, c_{E}\right)$ pairs from Fig. 1: $(4.0,-0.270),(6.0,-1.094),(8.0,-2.032)$, and $(10.0,-3.108)$. The resulting values of $c_{D}$ together with their errors are given in the right part of Fig. 2. In the left part theoretical predictions for the cross section as well as the data themselves are shown. It should be noted that at $70 \mathrm{MeV}$ and $135 \mathrm{MeV}$ in addition to statistical errors of data reported in [30] also a systematic error of 2\% [30] have been taken into account when calculating $\chi^{2}$. Since the resulting optimal value of $c_{D}$ slightly depends on the angular range of data used for calculating $\chi^{2}$, we provide in the right part also the information on that range. The values of $c_{D}$ found at $65 \mathrm{MeV}$ and 


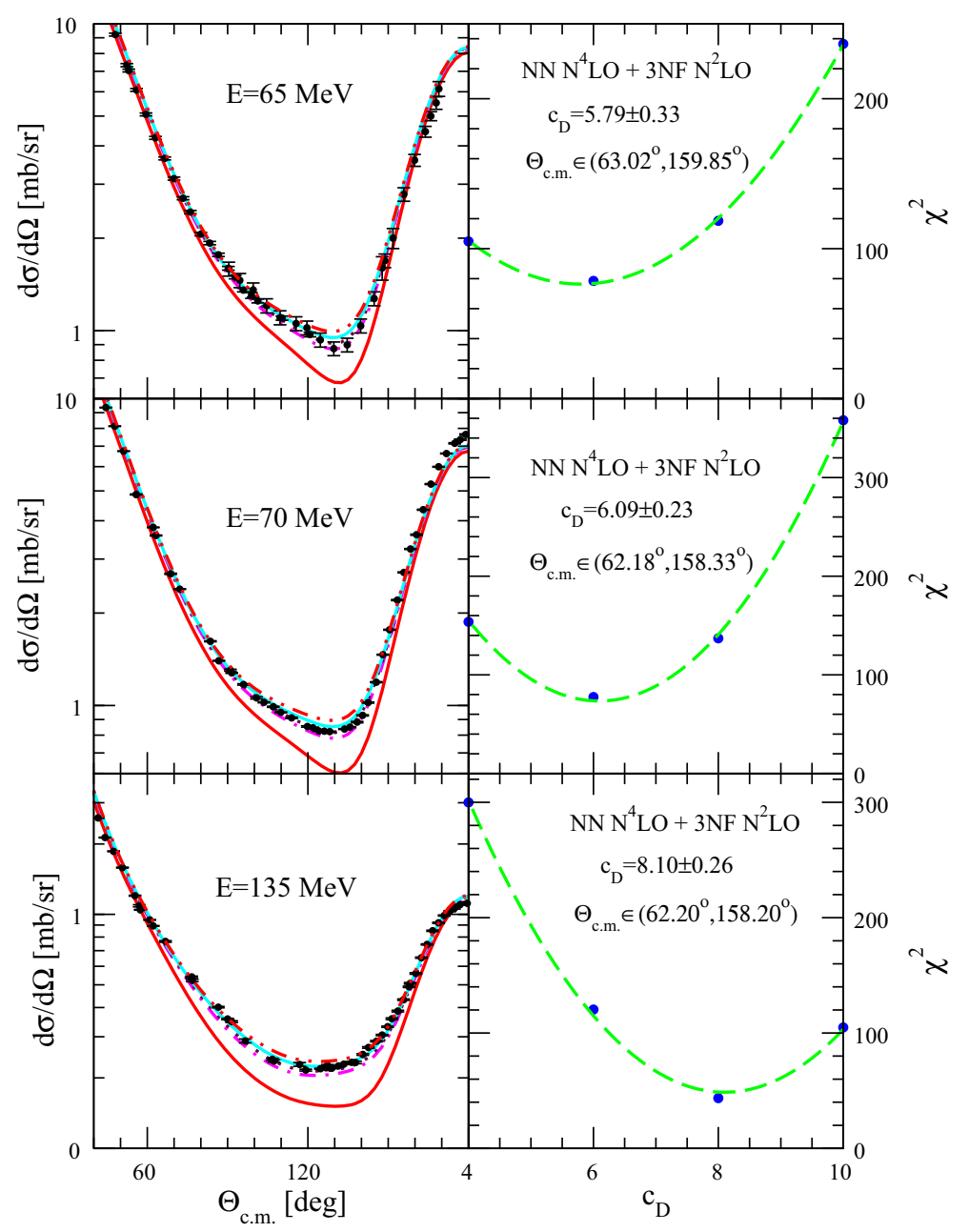

Fig. 2 The nd elastic scattering cross section at the incoming neutron laboratory energies $E=65$, 70, and $135 \mathrm{MeV}$. In the left part the solid (red) lines are predictions of the $\mathrm{N}^{4} \mathrm{LO}$ SCS NN potential with the regulator $R=0.9 \mathrm{fm}$. Combining that NN potential with $\mathrm{N}^{2} \mathrm{LO} 3 \mathrm{NF}$ with four different $\left(c_{D}, c_{E}\right)$ pairs of the strengths from the corelation line of Fig. 1 leads to results represented by different lines: dashed-dotted (magenta) $(4.0,-0.270)$, dotted (maroon) $(6.0,-1.094)$, solid (cyan) $(8.0,-2.032)$, and doubledotted-dashed (red) (10.0, -3.108). The (black) circles are pd data from Ref. [29] at $E=65 \mathrm{MeV}$, from Ref. [30] at $E=70 \mathrm{MeV}$, and from Ref. [30] at $E=135 \mathrm{MeV}$. In the right part the $\chi^{2}$ fits to the experimental data, in the angular region indicated at each energy, based on these four pairs of $\left(c_{D}, c_{E}\right)$ values, are shown by dashed (green) line together with the resulting optimal value of the strength $c_{D}$

$70 \mathrm{MeV}$ are compatible and agree within their arror bars. They are clearly smaller than the corresponding $c_{D}$ value obtained at $135 \mathrm{MeV}$. That difference originates from the growing importance of higher chiral order contributions to a $3 \mathrm{NF}$ with an increasing energy, which is effectively reflected in a larger value of $c_{D}$ at $135 \mathrm{MeV}$. This shows that in order to apply elastic Nd scattering cross section to fix the strengths $c_{D}$ and $c_{E}$ one should avoid too high energies. Using the correlation line from the upper part of Fig. 1 one gets for pairs of $\left(c_{D}, c_{E}\right)$ values and their errors $(5.79 \pm 0.33,-1.00 \pm 0.14)$ at $65 \mathrm{MeV}$ and $(6.09 \pm 0.23,-1.13 \pm 0.10)$ at $70 \mathrm{MeV}$.

It is interesting to note that at these three energies the elastic scattering cross section is reasonably well described when including the $\mathrm{N}^{2} \mathrm{LO} 3 \mathrm{NF}$. Our results also show that replacing ${ }^{2} a_{n d}$ by the cross section leads to a smaller by $\approx 1.5$ value of the $c_{D}$ strength what probably is connected with the fact that ${ }^{2} a_{n d}$ is an observable correlated with the ${ }^{3} \mathrm{H}$ binding energy. Due to the lack of this correlation and a richer set of cross section data, the second approach to fix strengths of $\mathrm{N}^{2} \mathrm{LO} 3 \mathrm{NF}$ contact terms seems to be preferable and can be done by also including the estimated truncation errors [17]. 


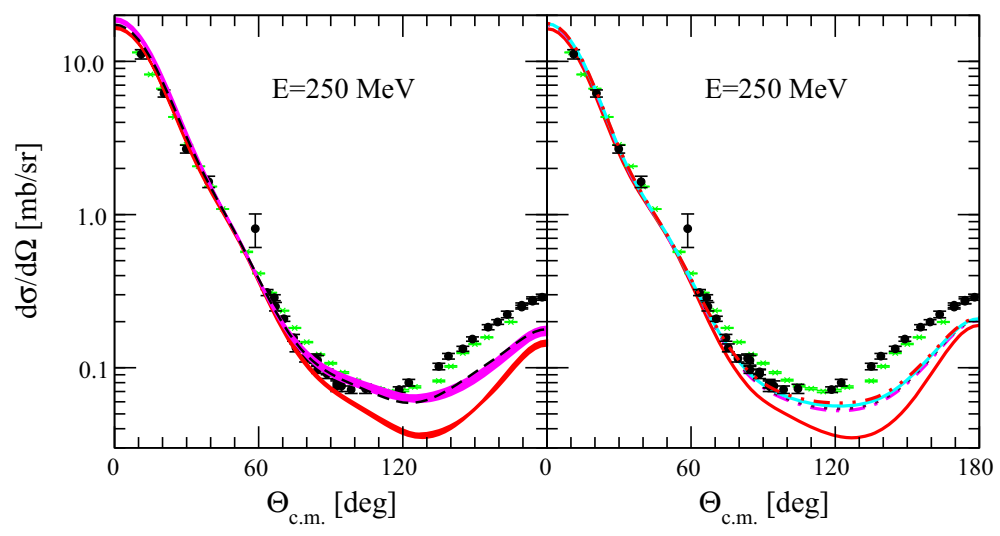

Fig. 3 The nd elastic scattering cross section at the incoming neutron laboratory energy $E=250 \mathrm{MeV}$. In the left part predictions of (semi-)phenomenological NN potentials (AV18, CD Bonn, Nijm1 and Nijm2) are shown by dark (red) band. Results obtained when these NN potentials are used together with the TM99 3NF are shown by a light (magenta) band. The dashed (black) line is the result of using a combination of the AV18 potential with the Urbana IX $3 \mathrm{NF}$. In the right part the different lines are predictions of the $\mathrm{N}^{4} \mathrm{LO}$ SCS NN potential with the regulator $R=0.9 \mathrm{fm}$ alone or combined with $\mathrm{N}^{2} \mathrm{LO} 3 \mathrm{NF}$ for four different $\left(c_{D}, c_{E}\right)$ strength pairs of the contact terms. For explanation of these lines see Fig. 2. In left and right parts the (black) dots are pd data from Ref. [38] and (green) x-ces are nd data from Ref. [39]

\section{Results for Elastic Scattering}

The good description of $\mathrm{Nd}$ elastic scattering cross section data up to about $130 \mathrm{MeV}$ when the $\mathrm{N}^{2} \mathrm{LO} 3 \mathrm{NF}$ is included in calculations resembles effects found with (semi-)phenomenological $2 \mathrm{~N}$ and $3 \mathrm{~N}$ forces $[4,28]$. Namely adding the TM99 or Urbana IX 3NF to (semi-)phenomenological NN potentials removed discrepancies between theory based on NN potentials only and the data, found in that energy region. At larger energies it turned out, however, that the inclusion of the $2 \pi$-exchange $3 \mathrm{~N}$ force models is unable to explain the growing discrepancies between data and theory as is exemplified in the left part of Fig. 3 for elastic Nd scattering at $250 \mathrm{MeV}$. A similar pattern of discrepancies is found when instead of (semi-)phenomenological interactions chiral SCS forces are used, as shown in the right part of Fig. 3. The astonishing similarity of (semi)phenomenological and chiral predictions can be traced back to the fact that the basic mechanism underlying these $3 N F$ 's is the $2 \pi$-exchange.

At low energies of the incoming neutron, the most interesting observable is the analyzing power $A_{y}$ for nd elastic scattering with polarized neutrons in the initial state. Theoretical predictions of (semi)phenomenological high-precision NN potentials fail to explain the experimental data for $A_{y}$. The data are underestimated by $\sim 30 \%$ in the region of the $A_{y}$ maximum which occurs at the c.m. angles $\Theta_{c . m} \approx 125^{\circ}$. Combining (semi-)phenomenological NN potentials with the $2 \pi$-exchange TM99 $3 N F$ model, removes approximately only half of the discrepancy to the data. That effect is, however, model dependent. If instead of the TM99 the Urbana IX 3NF is used, practically no effects on $A_{y}$ are found (see Fig. 4).

Consistency of chiral two- and three-nucleon forces promises to shed some light on the origin of this discrepancy. When instead of the (semi-)phenomenological forces chiral $\mathrm{N}^{4} \mathrm{LO} \mathrm{NN}$ interactions are used, the predictions for $A_{y}$ show the same behaviour. In particular a clear discrepancy between theory and data survives in the region of the $A_{y}$ maximum (dashed (red) lines in the right part of Fig. 4).

Combining the $\mathrm{N}^{4} \mathrm{LO}$ SCS chiral potential with the $\mathrm{N}^{2} \mathrm{LO} 3 \mathrm{NF}$ does not explain the low-energy $A_{y^{-}}$ puzzle. In Fig. 4 we show by (magenta) bands the results for $A_{y}$ based on four pairs of the $c_{D}$ and $c_{E}$ values, which reproduce the triton binding energy. It turns out that adding the $\mathrm{N}^{2} \mathrm{LO} 3 \mathrm{NF}$ only slightly improves the description of $A_{y}$. The effects found support that of the TM99 3NF, however, they are a factor of $\approx 2$ smaller in magnitude. The (magenta) band of predictions with $\mathrm{N}^{2} \mathrm{LO} 3 \mathrm{NF}$ included is very narrow in spite of the fact that the range of $c_{D}$ values $\left(c_{D}=4.0-10.0\right)$ (and the corresponding range of $\left.c_{E}\right)$ is quite large. This implies that low energy $A_{y}$ is insensitive to both contact terms of the $\mathrm{N}^{2} \mathrm{LO} 3 \mathrm{NF}$. In fact, this observable is very well known to be very sensitive only to ${ }^{3} P_{j} \mathrm{NN}$ force components [31] while contact terms act predominantly in the $\mathrm{S}$-waves. The small effect of $\mathrm{N}^{2} \mathrm{LO} 3 \mathrm{NF}$ found for $A_{y}$ may suggest that the $A_{y}$ puzzle is a problem of the poor knowledge about low-energy ${ }^{3} P_{j} \mathrm{NN}$ force components and not of $3 \mathrm{NF}$ effects. On the other hand, the theoretical uncertainty due to the truncation error of the chiral expansion for the $\mathrm{N}^{2} \mathrm{LO} N \mathrm{NN}$ and $\mathrm{N}^{2} \mathrm{LO} 3 \mathrm{NF}$ 


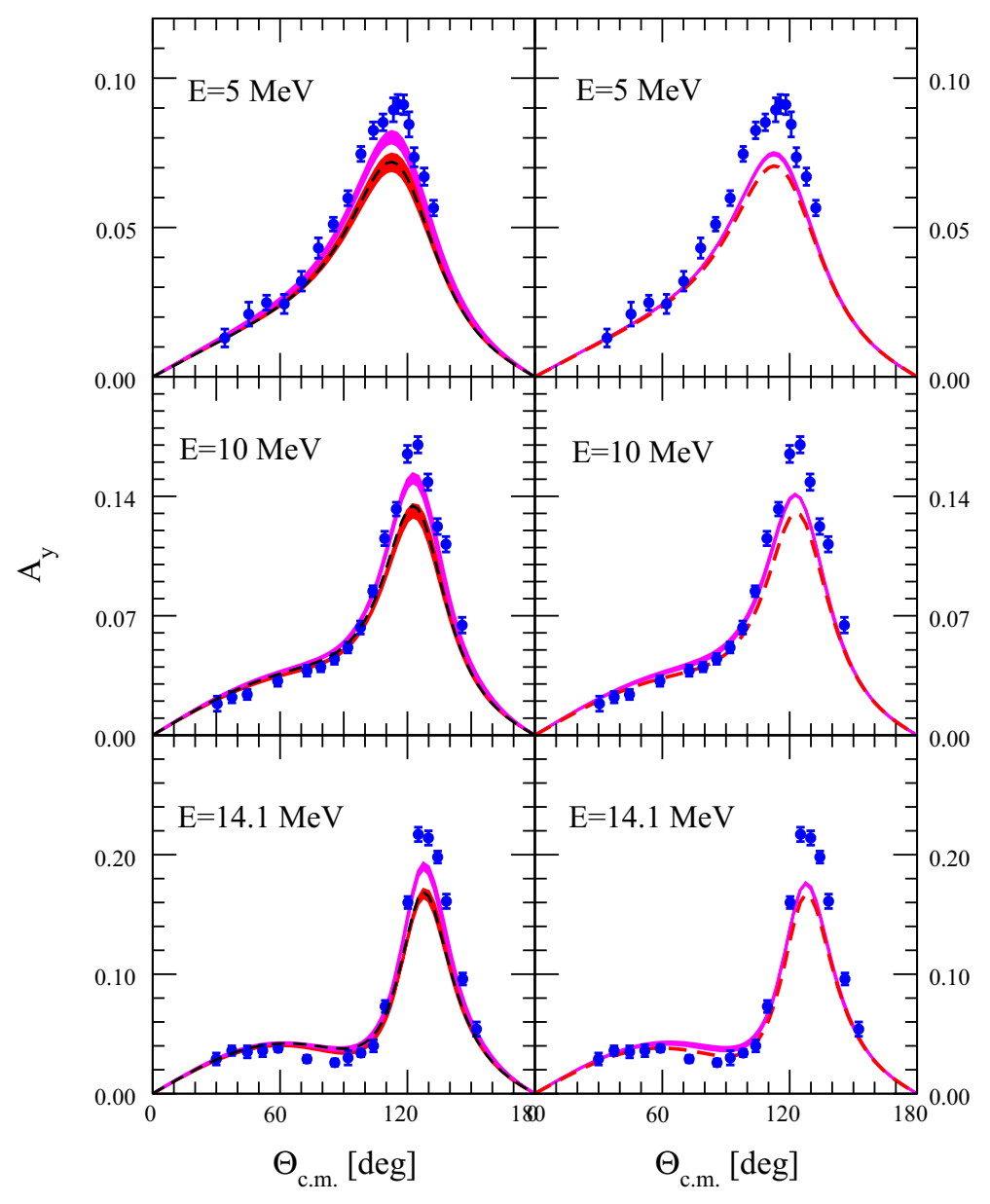

Fig. 4 The nd elastic scattering neutron analyzing power $A_{y}$ at the incoming neutron laboratory energies $E=5,10$, and 14.1 MeV. The predictions of (semi-)phenomenological NN potentials alone or combined with the TM99 and the Urbana IX 3NF's are shown in the left column by different bands and lines as explained in Fig. 3. In the right column the dashed (red) lines are predictions of the $\mathrm{N}^{4} \mathrm{LO}$ SCS NN potential with the regulator $R=0.9 \mathrm{fm}$. The bands (magenta) cover predictions obtained with that $\mathrm{N}^{4} \mathrm{LO} \mathrm{NN}$ potential combined with the $\mathrm{N}^{2} \mathrm{LO} 3 \mathrm{NF}$ for four $\left(c_{D}, c_{E}\right)$ pairs of strengths of its contact terms as explained in the caption of Fig. 2. The (blue) dots are nd data from Ref. [40] at $E=5 \mathrm{MeV}$, from Ref. [41] at $E=10 \mathrm{MeV}$, and from Ref. [42] at $E=14.1 \mathrm{MeV}$

combination was found to be comparable in magnitude with the observed deviation between $A_{y}$ predicitions and data [17]. Thus, before drawing final conclusions, $\mathrm{N}^{3} \mathrm{LO}$, and maybe even $\mathrm{N}^{4} \mathrm{LO}, 3 \mathrm{NF}$ effects for $A_{y}$ must be investigated.

With increasing incoming nucleon energy, $A_{y}$ should start to become dependent on contact terms and reveal sensitivity to $c_{D}$ and $c_{E}$ values. Indeed, as can be seen in the right part of Fig. 5 at higher energies the (magenta) band becomes broader, and beyond an intermediate region of energies around $60 \mathrm{MeV}$, where predicted effects of $\mathrm{N}^{2} \mathrm{LO} 3 \mathrm{NF}$ are very small and a good description of $A_{y}$ data is obtained, large discrepancies between pure $2 \mathrm{~N}$ theory and data start to develop. Again the pattern of discrepancies found for $A_{y}$ at these energies with chiral forces qualitatively resembles that obtained with (semi-)phenomenological interactions as shown in the left part of Fig. 5. A similar behaviour is revealed also for numerous other spin observables [4]. In addition it is the region of higher energies, where higher chiral order terms start to play an important role and short-range components of the $3 \mathrm{~N}$ force contribute, which presents a challenge for future applications of a $\mathrm{N}^{3} \mathrm{LO} 3 \mathrm{NF}$.

\section{Results for Breakup}

Among numerous kinematically complete configurations of the $\mathrm{Nd}$ breakup reaction the so-called symmetric space star (SST) and quasi-free scattering (QFS) configurations have attracted a special attention. The cross 


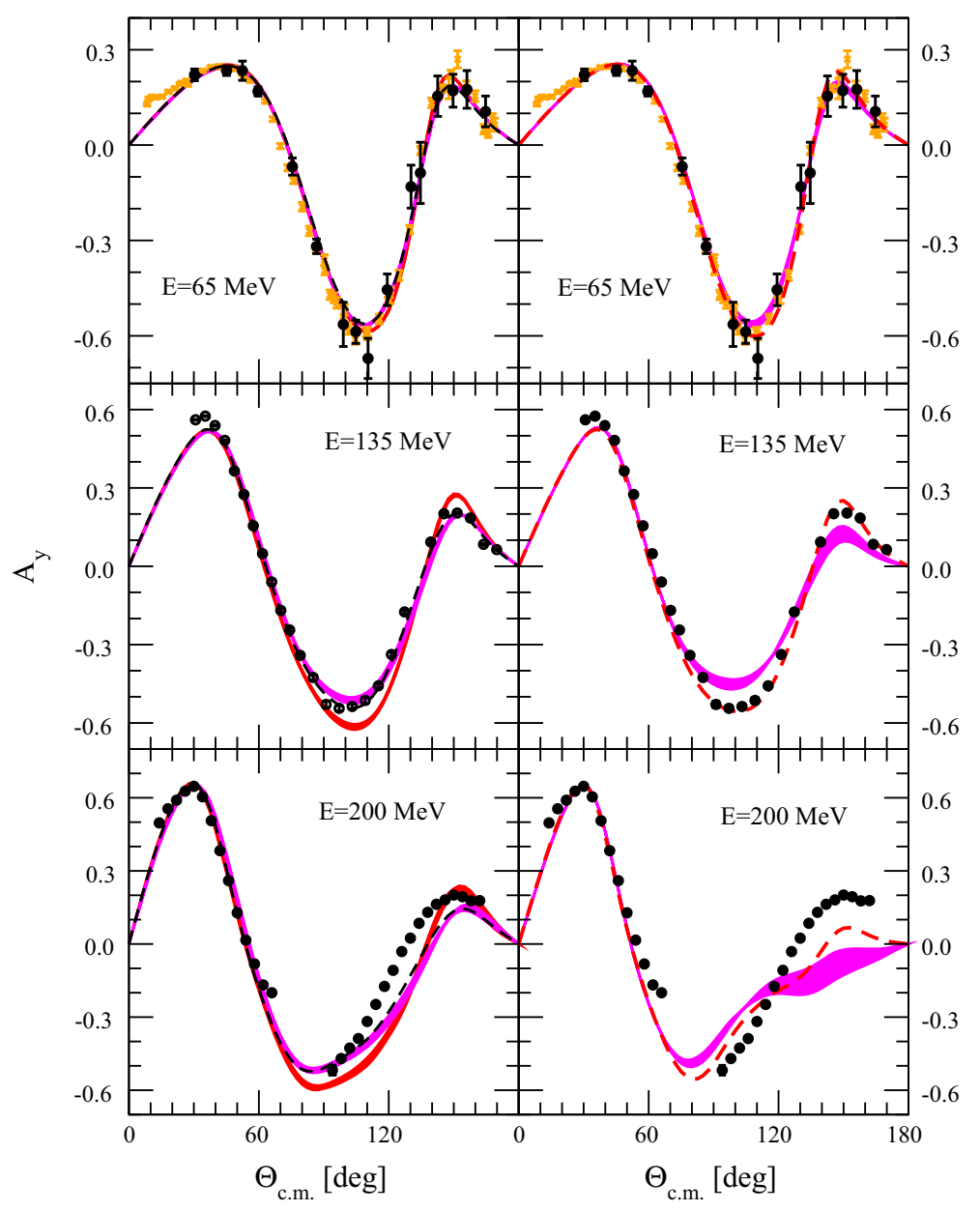

Fig. 5 The nd elastic scattering neutron analyzing power $A_{y}$ at the incoming neutron laboratory energies $E=65,135$, and $200 \mathrm{MeV}$. In the left column predictions of (semi-)phenomenological NN potentials alone or combined with the TM99 and the Urbana IX 3NF's are shown while in the right column predictions of the $\mathrm{N}^{4} \mathrm{LO}$ SCS NN potential with the regulator $R=0.9 \mathrm{fm}$ alone or combined with the $\mathrm{N}^{2} \mathrm{LO} 3 \mathrm{NF}$ for four $\left(c_{D}, c_{E}\right)$ pairs of strengths of the contact terms. For explanation of bands and lines see Fig. 4. At $E=65 \mathrm{MeV}$ the (black) dots are nd data from Ref. [43] and the (orange) x-ces are pd data from Ref. [29]. At $E=135 \mathrm{MeV}$ the (black) dots are pd data from Ref. [44]. At $E=200 \mathrm{MeV}$ the (black) dots are pd data from Ref. [45]

sections for these geometries are very stable with respect to the underlying dynamics and are dominated by the S-waves [32].

In Fig. 6, we compare predictions of the $\mathrm{N}^{4} \mathrm{LO}$ SCS chiral potential to the SST cross section data at two incoming nucleon energies $E=13 \mathrm{MeV}$ and $65 \mathrm{MeV}$. Given the good agreement at $65 \mathrm{MeV}$ with the experimental data of Ref. [33] as visualized in the right panel of Fig. 6, there is not much room for 3NF effects for this observable. Indeed, adding $\mathrm{N}^{2} \mathrm{LO} 3 \mathrm{NF}$ only slightly increases the cross section which practically does not depend on the strengths of that $3 \mathrm{NF}$ contact terms. At $13 \mathrm{MeV}$, the nd and pd breakup data are far away from the theory. The nd data set shows a significant disagreement with our theoretical results. The pd data set shown in Fig. 6 is supported by other SST pd breakup measurements [34] in a similar energy range. The calculations of the pd breakup with inclusion of the pp Coulomb force [35] revealed only very small Coulomb force effects for this configuration. Since, at that energy, the SST configuration is practically dominated by the S-wave NN force components, the big difference between pd and nd data seems to indicate significant charge-symmetry breaking in the ${ }^{1} S_{0} \mathrm{NN}$ partial wave. We expect difficulties with explaining the large difference between the nd and pd data sets by the inclusion of a $3 \mathrm{NF}$ without introducing large charge symmetry breaking interactions. Furthermore, the discrepancy between the theory and experimental pd data is puzzling. The inclusion of the chiral $\mathrm{N}^{2} \mathrm{LO}$ 3NF does not affect the results for the SST configuration cross sections at this energy at all, leaving a big discrepancy to both nd and pd data. 


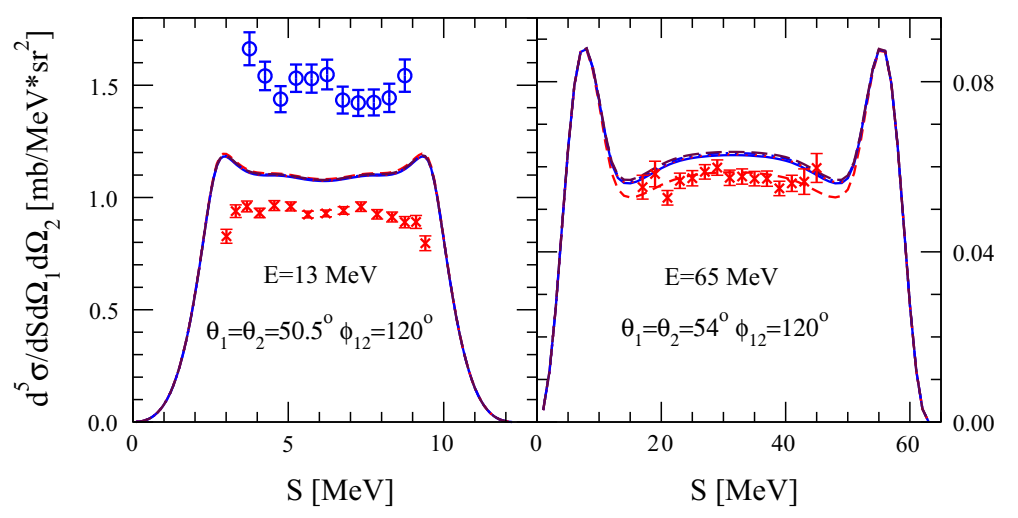

Fig. 6 The nd breakup five-fold differential cross section in the SST complete geometry at the incoming neutron laboratory energies $E=13 \mathrm{MeV}$ and $E=65 \mathrm{MeV}$, shown as a function of arc-length of the kinematical S-curve [19]. The dashed (red) lines are predictions of the $\mathrm{N}^{4} \mathrm{LO} S C S \mathrm{NN}$ potential with the regulator $R=0.9 \mathrm{fm}$. Combining that NN potential with the $\mathrm{N}^{2} \mathrm{LO}$ $3 \mathrm{NF}$ with four different strengths $\left(c_{D}, c_{E}\right)$ of the contact terms from the corelation line of Fig. 1 leads to results shown by different lines: solid (blue) $(4.0,-0.270)$, dotted (red) $(6.0,-1.094)$, double-dotted-dashed (blue) $(8.0,-2.032)$, and dashed (maroon) $(10.0,-3.108)$. At $E=13 \mathrm{MeV}$ the (blue) circles are nd data from Ref. [46] and the (red) x-ces are pd data from Ref. [47]. At $E=65 \mathrm{MeV}$ the (red) $\mathrm{x}$-ces are pd data from Ref. [33]

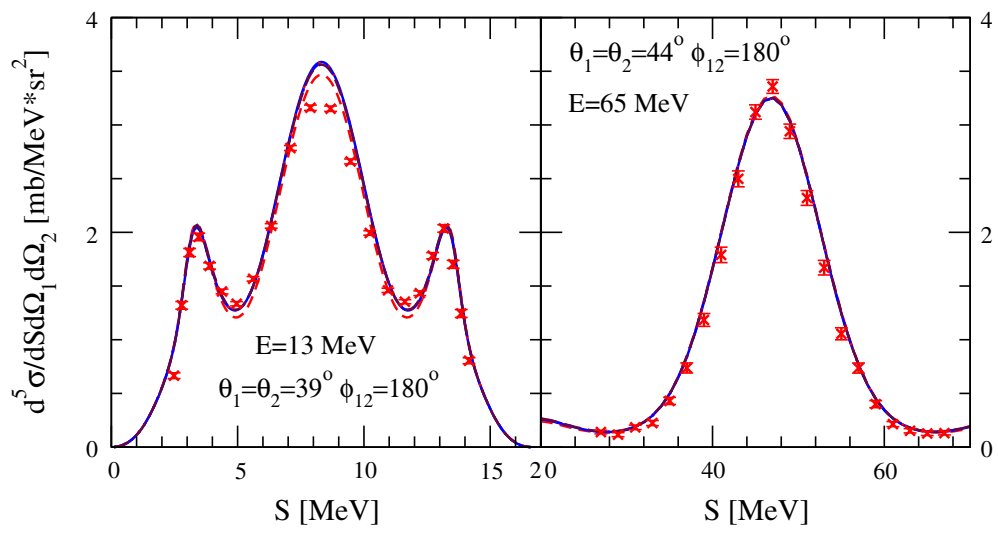

Fig. 7 The nd breakup five-fold differential cross section in the QFS complete geometry at the incoming neutron laboratory energies $E=13 \mathrm{MeV}$ and $E=65 \mathrm{MeV}$, shown as a function of arc-length of the S-curve. Lines are the same as in Fig. 6 . The (red) x-ces are pd data from Ref. [47] at $E=13 \mathrm{MeV}$ and from Ref. [48] at $E=65 \mathrm{MeV}$

For the pp QFS geometry, we show in Fig. 7 the predictions based on the $\mathrm{N}^{4} \mathrm{LO}$ chiral potentials at $E=13 \mathrm{MeV}$ and $65 \mathrm{MeV}$, together with the available pd breakup data. The theoretical predictions agree well with the pd breakup data and for that configuration practically no effects of the $\mathrm{N}^{2} \mathrm{LO} 3 \mathrm{NF}$ are present.

\section{Summary and Conclusions}

We applied the semilocal coordinate-space regularized $\mathrm{N}^{4} \mathrm{LO}$ chiral $\mathrm{NN}$ potential combined with the $\mathrm{N}^{2} \mathrm{LO}$ $3 \mathrm{NF}$, regularized in the same way, to reactions in the $3 \mathrm{~N}$ continuum. We checked that using elastic Nd scattering cross section data as an additional (to the ${ }^{3} \mathrm{H}$ binding energy) $3 \mathrm{~N}$ observable provides an efficient tool to fix the strengths $c_{D}$ and $c_{E}$ of the $\mathrm{N}^{2} \mathrm{LO} 3 \mathrm{NF}$ contact terms. However, the application of this tool should be restricted to the region of the incoming nucleon laboratory energies around $\approx 60 \mathrm{MeV}$, where $3 \mathrm{NF}$ effects start to appear in the cross section.

The application of that particular combination of $\mathrm{NN}$ and $3 \mathrm{~N}$ forces provides at higher energies effects for the elastic scattering cross section which are very similar to those obtained with (semi-)phenomenological interactions. For the low-energy nucleon analyzing power $A_{y}$ the application of consistent chiral forces supports predictions of the TM99 3NF, however, the resulting effects are smaller in magnitude by a factor of $\approx 2$. Thus the $\mathrm{N}^{2} \mathrm{LO} 3 \mathrm{NF}$ is unable to explain the low-energy $A_{y}$ puzzle. That $3 \mathrm{NF}$ is also incapable of explaining discrepancies between data and theory for $13 \mathrm{MeV}$ SST complete breakup configuration. At higher energies 
and for numerous elastic scattering spin observables a complex pattern of discrepancies to data was found, similar to that revealed in previous investigations with (semi-)phenomenological $\mathrm{NN}$ and $3 \mathrm{~N}$ forces. The chiral results are sensitive to the magnitude of contact terms. It points to a growing importance of short-range components of a $3 \mathrm{NF}$ at higher energies and calls for an application of the $\mathrm{N}^{3} \mathrm{LO} 3 \mathrm{NF}$, in which numerous new components contribute [36,37].

Acknowledgements This study has been performed within Low Energy Nuclear Physics International Collaboration (LENPIC) project and was supported by the Polish National Science Center under Grant Nos. 2016/22/M/ST2/00173 and 2016/21/D/ST2/01120 and by the BMBF (Grant No. 05P15PCFN1). The numerical calculations were performed on the supercomputer cluster of the JSC, Jülich, Germany.

Open Access This article is distributed under the terms of the Creative Commons Attribution 4.0 International License (http:// creativecommons.org/licenses/by/4.0/), which permits unrestricted use, distribution, and reproduction in any medium, provided you give appropriate credit to the original author(s) and the source, provide a link to the Creative Commons license, and indicate if changes were made.

\section{References}

1. R.B. Wiringa et al., Accurate nucleon-nucleon potential with charge-independence breaking. Phys. Rev. C 51, 38-51 (1995)

2. R. Machleidt, High-precision, charge-dependent Bonn nucleon-nucleon potential. Phys. Rev. C 63, 024001-1-32 (2001)

3. V.G.J. Stoks et al., Construction of high-quality NN potential models. Phys. Rev. C 49, 2950-2963 (1994)

4. H. Witała et al., Nd elastic scattering as a tool to probe properties of 3N forces. Phys. Rev. C 63, 024007-1-12 (2001). and references therein

5. S.A. Coon, H.K. Han, Reworking the Tucson-Melbourne three-nucleon potential. Few Body Syst. 30, 131-141 (2001)

6. B.S. Pudliner et al., Quantum Monte Carlo calculations of nuclei with $A<7$. Phys. Rev. C 56, 1720-1750 (1997)

7. E. Epelbaum et al., The two-nucleon system at next-to-next-to-next-to-leading order. Nucl. Phys. A 747, 362-424 (2005)

8. E. Epelbaum, Few nucleon forces and systems in chiral effective field theory. Prog. Part. Nucl. Phys. 57, 654-741 (2006)

9. R. Machleidt, D.R. Entem, Chiral effective field theory and nuclear forces. Phys. Rep. 503, 1-76 (2011)

10. H. Witała et al., Calculations of three-nucleon reactions with $\mathrm{N}^{3} \mathrm{LO}$ chiral forces: achievements and challenges. J. Phys. G Nucl. Part. Phys. 41, 094011-1-21 (2014)

11. E. Epelbaum et al., Improved chiral nucleon-nucleon potential up to next-to-next-to-next-to-leading order. Eur. Phys. J. A 51, 53-81 (2015)

12. E. Epelbaum et al., Precision nucleon-nucleon potential at fifth order in the chiral expansion. Phys. Rev. Lett. 115, 122301-1-5 (2015)

13. S. Binder et al., Few-nucleon systems with state-of-the-art chiral nucleon-nucleon forces. Phys. Rev. C 93, 044002-1-6 (2016)

14. S. Binder et al., Few- and many-nucleon systems with semilocal coordinate-space regularized chiral nucleon-nucleon forces. Phys. Rev. C 98, 014002-1-30 (2018)

15. P. Reinert, H. Krebs, E. Epelbaum, Semilocal momentum-space regularized chiral two-nucleon potentials up to fifth order. Eur. Phys. J. A 54, 86-1-49 (2018)

16. E. Epelbaum et al., Three-nucleon forces from chiral effective field theory. Phys. Rev. C 66, 064001-1-17 (2002)

17. E. Epelbaum et al., Few- and many-nucleon systems with semilocal coordinate-space regularized chiral two- and three-body forces. arXiv:1807.02848 [nucl.th]

18. H. Witała et al., Elastic scattering and break-up processes in the n-d system. Few-Body Syst. 3, 123-134 (1988)

19. W. Glöckle et al., The three-nucleon continuum: achievements, challenges and applications. Phys. Rep. 274, 107-285 (1996)

20. D. Hüber et al., How to include a three-nucleon force into Faddeev equations for the $3 \mathrm{~N}$ continuum: a new form. Acta Phys. Pol. B 28, 1677-1685 (1997)

21. K. Hebeler et al., Efficient calculation of chiral three-nucleon forces up to $\mathrm{N}^{3} \mathrm{LO}$ for ab initio studies. Phys. Rev. C 91, 044001-1-9 (2015)

22. A. Nogga, D. Hüber, H. Kamada, W. Glöckle, Triton binding energies for modern NN forces and the $\pi-\pi$ exchange three-nucleon force. Phys. Lett. B 409, 19-25 (1997)

23. D. Gazit, S. Quaglioni, P. Navratil, Three-nucleon low-energy constants from the consistency of interactions and currents in chiral effective field theory. Phys. Rev. Lett. 103, 102502-1-4 (2009)

24. H. Witała, A. Nogga, H. Kamada, W. Glöckle, J. Golak, R. Skibiński, Modern nuclear force predictions for the neutrondeuteron scattering length. Phys. Rev. C 68, 034002-1-8 (2003)

25. K. Schoen et al., Precision neutron interferometric measurements and updated evaluations of the np and nd coherent neutron scattering lengths. Phys. Rev. C 67, 044005-1-21 (2003)

26. W. Glöckle, The Quantum Mechanical Few-Body Problem (Springer, Berlin, 1983)

27. C. Howell et al., Resolution of discrepancy between backward angle cross-section data for neutron-deuteron elastic scattering. Few-Body Systems 16, 127-142 (1994)

28. H. Witała et al., Cross section minima in elastic Nd scattering: possible evidence for three-nucleon force effects. Phys. Rev. Lett. 81, 1183-1186 (1998)

29. H. Shimizu et al., Analyzing powers and cross sections in elastic scattering at $65 \mathrm{MeV}$. Nucl. Phys. A 382, 242-254 (1982)

30. K. Sekiguchi et al., Complete set of precise deuteron analyzing powers at intermediate energies: comparison with modern nuclear force predictions. Phys. Rev. C 65, 034003-1-16 (2002)

31. W. Tornow, H. Witała, Evaluation of the three-nucleon analyzing power puzzle. Nucl. Phys. A 637, $280-292$ (1998) 
32. H. Witała, W. Glöckle, On the discrepancies in the low-energy neutron-deuteron breakup. J. Phys. G Nucl. Part. Phys. 37, 064003-1-11 (2010)

33. J. Zejma et al., Cross sections and analyzing powers $A_{y}$ in the breakup reaction ${ }^{2} H(\vec{p}, p p) n$ at $65 \mathrm{MeV}$ : star configurations. Phys. Rev. C 55, 42-56 (1997)

34. K. Sagara, Experimental investigations of discrepancies in three-nucleon reactions. Few-Body Syst. 48, 59-108 (2010)

35. A. Deltuva et al., Momentum-space description of three-nucleon breakup reactions including the Coulomb interaction. Phys. Rev. C 72, 054004-1-12 (2005)

36. V. Bernard, E. Epelbaum, H. Krebs, U.-G. Meißner, Subleading contributions to the chiral three-nucleon force: long-range terms. Phys. Rev. C 77, 064004-1-13 (2008)

37. V. Bernard, E. Epelbaum, H. Krebs, U.-G. Meißner, Subleading contributions to the chiral three-nucleon force. II. Short-range terms and relativistic corrections. Phys. Rev. C 84, 054001-1-12 (2011)

38. K. Hatanaka et al., Cross section and complete set of proton spin observables in $\vec{p} d$ elastic scattering at $250 \mathrm{MeV}$. Phys. Rev. C 66, 044002-1-10 (2002)

39. Y. Maeda et al., Differential cross section and analyzing powerc measurements for $\vec{n} d$ elastic scattering at $248 \mathrm{MeV}$. Phys. Rev. C 76, 014004-1-13 (2007)

40. W. Tornow et al., The low-energy neutron-deuteron analyzing power and the ${ }^{3} P_{0,1,2}$ interactions of nucleon-nucleon potentials. Phys. Lett. B 257, 273-277 (1991)

41. W. Tornow, C.R. Howell, R.C. Byrd, R.S. Pedroni, R.L. Walter, Analyzing power measurements for $\left({ }^{2} H\left(n_{p o l}, n\right)^{2} H\right)$ scattering at $10 \mathrm{MeV}$ compared to few-nucleon calculations and data for $\left({ }^{2} H\left(p_{p o l}, p\right)^{2} H\right)$ scattering. Phys. Rev. Lett. 49, $312-315(1982)$

42. W. Tornow, R.C. Byrd, C.R. Howell, R.S. Pedroni, R.L. Walter, Measurements of analyzing power for ${ }^{2} H(\vec{n}, n)^{2} H$ scattering at $14.1 \mathrm{MeV}$ and comparisons to ${ }^{2} H(\vec{p}, p)^{2} H$. Phys. Rev. C 27, 2439-2442 (1983)

43. H. Rühl et al., Analyzing power in elastic scattering at $67 \mathrm{MeV}$. Nucl. Phys. A 524, 377-390 (1991)

44. K. Ermisch et al., Systematic investigation of three-nucleon force effects in elastic scattering of polarized protons from deuterons at intermediate energies. Phys. Rev. C 71, 064004-1-21 (2005)

45. B.V. Przewoski et al., Analyzing powers and spin correlation coefficients for $p+d$ elastic scattering at 135 and $200 \mathrm{MeV}$. Phys. Rev. C 74, 064003-1-21 (2006)

46. H.R. Setze et al., Verification of the space-star anomaly in nd breakup. Phys. Lett. B 388, 229-234 (1996)

47. G. Rauprich et al., Study of the kinematically complete breakup reaction ${ }^{2} H(\vec{p}, p p) n$ at $E_{p}=3 \mathrm{MeV}$ with polarized protons. Nucl. Phys. A 535, 313-330 (1991)

48. M. Allet et al., Proton-induced deuteron breakup at $E_{l a b}^{p}=65 \mathrm{MeV}$ in quasi-free scattering configurations. Few-Body Syst. 20, 27-40 (1996)

Publisher's Note Springer Nature remains neutral with regard to jurisdictional claims in published maps and institutional affiliations. 